\title{
Robot Navigation based on the Mapping of Coarse Qualitative Route Descriptions to Route Graphs
}

\author{
Christian Mandel*, Udo Frese ${ }^{\dagger}$, Thomas Röfer ${ }^{\ddagger}$ \\ Department of Computer Science \\ Universität Bremen \\ P.O.Box 330440 \\ Germany - 28334 Bremen \\ Email: $\left\{{ }^{*} \mathrm{cman},{ }^{\dagger}\right.$ ufrese, ${ }^{\dagger}$ roefer $\} @$ informatik.uni-bremen.de
}

\begin{abstract}
This paper describes the use of natural language route descriptions in the mobile robot navigation domain. Guided by corpus analysis and earlier work on coarse qualitative route descriptions, we decompose instructions given by humans into sequences of imprecise route segment descriptions. By applying fuzzy rules for the involved spatial relations and actions, we construct a search tree that can be searched in a depth-first branchand-bound manner for the most probable goal configuration w.r.t. the global workspace knowledge of the robot. The applicability of our approach is shown by a real-world experiment where an operator instructs his automated wheelchair to navigate in an office-like environment.
\end{abstract}

\section{INTRODUCTION}

Since robots have found their way from sealed work-stations in factories to populated places such as museum halls, railway stations, or hospitals, it is clear that sharing an environment with human beings requires suitable means of communication between these two groups. Apart from safety issues, appropriate communication becomes even more important if man and machine collaborate in order to fulfil a task. Service robots serve as a good example for these needs.

In our case, the autonomous wheelchair "Rolland" [1] is intended to assist its operator in various navigation tasks. The most natural application scenario allows the wheelchair-bound person to ask the device to go to a desired goal location e.g. "Please bring me to the kitchen.". In the case that the system's map of the environment has never been annotated with the symbol "kitchen", a clarifying utterance such as "Go down the corridor and take the second door to the left." could recover from potential failure. The work presented in this paper applies to task descriptions of the latter form.

We begin in section II with a brief overview of approaches to communicate qualitative task descriptions to a robot. Section III continues with the description of our experimental platform and accounts for the utilized prerequisites from the robotics domain, e.g. data structures for global world knowledge, self localization, and the local navigation method applied. In section IV we present the formalization of our socalled Coarse Route Descriptions (CRD). As an extension to the work in [2], they are based on the analysis of corpora which were aquired during an experiment where about 27 subjects had to give route descriptions to a designated goal, while operating the autonomous wheelchair Rolland [3]. In section $\mathrm{V}$ we present our approach to mapping CRDs to the global environment map. Commencing with the global pose of the system, we apply fuzzy rules for the spatial relations that describe the involved sub-goals, as well as subsequent actions which mostly comprise reorientations along the route to the goal. In section VI we conclude with the presentation of experimental results that where acquired by the execution of an a priori given coarse qualitative route description.

\section{RELATED WORK}

Current research in human-robot interaction (HRI) comprises a vast spectrum of applied interface techniques. Thrun gives in [4] a broad survey of the state of the art and divides current approaches into direct and indirect interaction. Whereas direct communication means that the robot and its operator are able of initiating a dialogue act, indirect communication solely allows the operator to give commands, possibly followed by a response from the robot. Another distinction of HRI is given in [5], where the authors contrast the so-called "Front-End" approach that completely specifies the task to be performed by the robot against an incremental approach. The latter one is based on the decomposition of task descriptions into elementary actions, thus allowing for dynamic changes in the execution phase.

From the perspective of instructed robot navigation, different modalities in HRI have been established. Kyriacou et.al. describe in [6] a verbally instructed robot that executes a given route description in an artificial miniature town. Encouraged by corpora analysis they transfer a given route description into a list of action chunks accompanied by spatial relations and landmarks. During the execution phase the system builds on computer vision, e.g. for the recognition of referenced road features. We can anticipate that this approach differs from our work in that it solely relies on local sensor maps instead of exploiting prior available global knowledge. Hence, the comparison of both approaches is a good example for the contrasting incremental, resp. front-end approaches.

Unlike natural language driven interfaces, Chronis et.al. use sketched route maps for communicating with a mobile robot [7]. Hand-drawn landmarks that are derived from closed polygons are enriched by spatial relations that indicate their position w.r.t. the sketched route. The resulting Qualitative 
Landmark States (QLS) are finally matched against the sensorial input in order to keep the robot on track.

\section{EXPERIMENTAL PlatForm AND PREREQUisites FROM THE ROBOTICS DOMAIN}

Our experimental platform Rolland III is the batterypowered wheelchair Meyra Champ 1.594. In contrast to its predecessor that was equipped with a ring of sonar sensors, and later on with a single backward facing laser range finder [8], Rolland III is equipped with two laser scanners mounted at ground level, which allow for scanning beneath the feet of a human operator. As an additional sensor device, the system provides two incremental encoders which measure the rotational velocity of two independently actuated wheels. Please note that the provided information needed for dead reckoning is highly imprecise due to variable wheel diameter and slippage.

\section{A. Local Navigation and Obstacle Avoidance}

The cruicial factor for the applicability of a mobile robot system in a real world scenario is its ability to deal with dynamic and unforeseen obstacles. To do so, almost all approaches use periodic sensor measurements in order to maintain a map of the vicinity of the robot. Using this local map, they either compute geometric paths or apply reactive behaviours in order to reach a locally defined goal while avoiding perceived obstacles. Common examples are the Dynamic Window Approach (DWA) [9], the Virtual Force Field Method (VFFM) [10], Nearness Diagram Navigation (NDN) [11] and many others.

The basic DWA, as an example for path planning algorithms, turned out to be not suitable for our needs, because it only checks single arcs with fixed curvature in every cycle of computation. Although this approach is sufficient for circular robots being able to turn around their midpoint while entering a narrow passage, it does not model a necessary haul-off movement needed by vehicles turning around the center of their rear axle. A similar problem arises when employing a purely behaviour-based approach like the VFFM. Here the robot is modeled as a particle influenced by the sum of repulsive forces from the obstacle points and an attractive force starting from the desired goal. It is obvious that one cannot achieve shunting behaviours using this technique. Minguez et al. proposed a method for incorporating the kinematics and the shape of a robot in behaviour-based techniques [12]. The authors exemplified their approach by applying the VFFM and NDN in their so-called Ego-KinoDynamic Space, and thereby explicitly taking account for the contour and the kinematic properties of the used robot. Despite this refinement, the overall algorithm still looks only one curve ahead, leaving the problem of haul-off movements untreated.

Starting from these insights we decided to employ a geometric path planner using cubic Bezier curves, since they are able to connect two given points while accounting for a desired curve progression and for directional requirements in the start point and end point. Considering the work of Hwang et al.

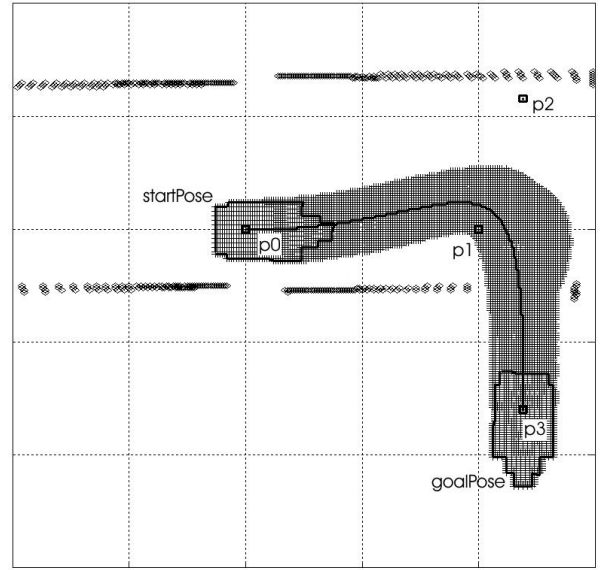

Fig. 1. Occupancy grid including cubic Bezier curve-based path, connecting startPose and goalPose. Control points $\vec{p}_{1}$ and $\vec{p}_{2}$ are located on straight lines that i) pass through $\vec{p}_{0}$ resp. $\vec{p}_{3}$ and ii) are aligned to the orientation of startPose resp. goalPose.

[13] which gives a broad overview on approaches using this type of curve, we will now sketch our basic algorithm. Given the current pose of the wheelchair startPose $=\left(x_{s}, y_{s}, \theta_{s}\right)$ and the desired target goalPose $=\left(x_{g}, y_{g}, \theta_{g}\right)$, we search the space of cubic Bezier curves for paths that

i) connect $\vec{p}_{0}=\left(x_{s}, y_{s}\right)$ with $\vec{p}_{3}=\left(x_{g}, y_{g}\right)$,

ii) are smoothly aligned with $\theta_{s}$ in $\vec{p}_{0}$ and with $\theta_{g}$ in $\vec{p}_{3}$,

iii) are obstacle free in the sense that a contour of the robot shifted tangentially along the path does not intersect with any obstacle point from a given occupancy grid $O G$.

Equation (1) describes a cubic Bezier curve, connecting the points $\vec{p}_{0}$ and $\vec{p}_{3}$, at a given arc length $t \in[0 . .1]$. In order to unequivocally determine the characteristics of the curve, we still have to chose the control points $\vec{p}_{1}$ and $\vec{p}_{2}$ such that we fulfil requirements ii) and iii).

$$
\begin{aligned}
\vec{p}(t) & =\vec{a} t^{3}+\vec{b} t^{2}+\vec{c} t+\vec{p}_{0}, t \in[0 . .1] \\
\text { with } \vec{c} & =3\left(\vec{p}_{1}-\vec{p}_{0}\right), \\
\vec{b} & =3\left(\vec{p}_{2}-\vec{p}_{1}\right)-\vec{c}, \\
\vec{a} & =\vec{p}_{3}-\vec{p}_{0}-\vec{b}-\vec{c}
\end{aligned}
$$

The computation of the free parameters $\vec{p}_{1}$ and $\vec{p}_{2}$, as can be seen in (2), spans the search space over the cubic Bezier curves, whose solution is intended to solve our path planing problem.

$$
\begin{aligned}
& \vec{p}_{1}\left(l_{1}\right)=\vec{p}_{0}+l_{1} \overrightarrow{\left(\cos \left(\theta_{s}\right), \sin \left(\theta_{s}\right)\right)}, l_{1} \max >l_{1}>0 \\
& \vec{p}_{2}\left(l_{2}\right)=\vec{p}_{3}-l_{2} \overrightarrow{\left(\cos \left(\theta_{g}\right), \sin \left(\theta_{g}\right)\right)}, l_{2} \max >l_{2}>0
\end{aligned}
$$

Fig. 1 illustrates the result of a single path planning cycle. It shows the occupancy grid including the integral of former sensor measurements along with the current state of the robot startPose and the desired target goalPose. The solid drawn cubic Bezier curve has been chosen as a solution in fulfillment 
TABLE I

EBNF Formalization of COARSE ROUTE DESCRIPTIONS. FOR THE SAKE OF COMPACTNESS WE OMIT THE DEFINITION OF CURSIVE PRINTED

NON-TERMINALS.

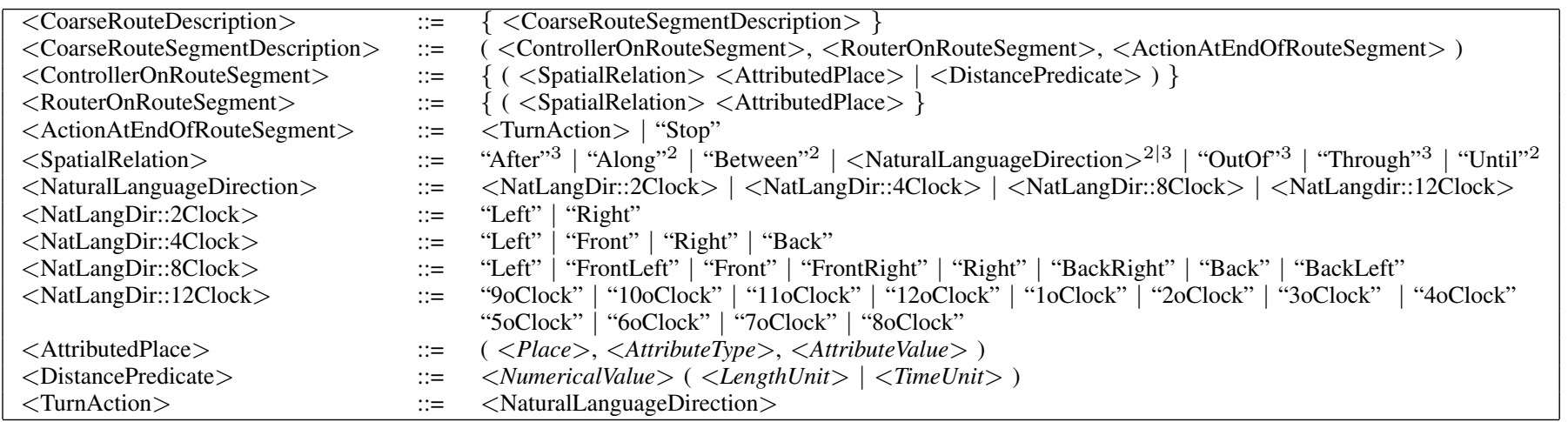

of requirements i) - iii) that minimizes the time of travel. The upper velocity-bound of the robot at point $\vec{p}(t)$ is therefore determined by the minimal distance between the robot-contour tangentially located at $\vec{p}(t)$ to any obstacle-point, and the curvature $c(t)$.

\section{B. Global Localization}

As an intermediate step before using already available topological information for localization, a Monte-CarloLocalization method was implemented that is based on the self-locator used by the German RoboCup Team [14]. To establish hypotheses of the current location of the wheelchair, particles are drawn from a pre-computed table that indexes the global environment by the area of the scan perceived from a certain position. In addition, only distance measurements resulting from flat surfaces i.e. from segmented lines are used to determine the current position. Thereby, persons standing around are ignored. The number of particles drawn from observations depends on how good the actual sensor measurements match the expected measurements from the positions of particles [15].

\section{RouteGraph as Data Structure for Global World Knowledge}

In order to solve high level tasks such as the interpretation of coarse qualitative route descriptions, one needs an appropriate global data structure that meets a list of requirements. Beside the adequate representation of navigable space, it has to be extendible by annotations that describe landmarks of interest. These annotations could be given either by humans or by automatic feature detectors. After all, it has to provide an interface that supplies basic spatial queries as a foundation for the evaluation of more elaborated spatial tasks. This interface is described in section $\mathrm{V}$.

In our work we apply a graph structured representation, i.e. the Route Graph, to do the job sketched above. In the route graph taxonomy, broadly described in [16] and [17], a route graph representing the whole workspace of the robot consists of several Route Graph Layers each of a different Kind. At several Places which represent distinguishable robot poses, the route graph layers are connected via Transitions, depicting a correspondance between places inside different kinds of map representation. On the other hand, places can be connected by Route Segments in a single route graph layer, stating that the robot can go from one place to the other. Concatenation of these route segments results in Routes, describing complex navigation tasks.

Our ongoing work is mainly focused on the topological layer of the route graph e.g. the analysis of its use in self localization tasks. The topological layer describes a network of places and route segments with maximal clearance to the surrounding obstacles, based on Voronoi diagrams. Our framework implemented so far is able to compute this description either from local sensor-based grid maps, or from a preexisting global grid map derived from a CAD blueprint.

\section{Formalization of CoARse Route Descriptions GIVEN VIA NATURAL LANGUAGE}

In this section we lay out the concept of interpreting coarse qualitative route descriptions, usually given by humans to other agents. The main idea, borrowed from [2], states that the concerned route descriptions can be segmented into four categories: starting-point, path/progression, reorientation and goal. Following this insight, the authors develop a route description as a sequence of tupels of the following form:

$$
<[\{\text { controlmarks }\} \text { router }] \text { reorientation }>
$$

Starting at the current pose of the agent, controlmarks describe distinctive landmarks along a route segment without directional changes. By reaching a router that depicts a place where a directional change may occur, a reorientation stands for the directional instruction that aligns the agent to the new route segment. The following route segments are encoded in the same way with the only difference that the the final router represents the goal of the whole route description. Before we describe miscellaneous enhancements of this formalization, we take a brief look at some corpus examples in order to justify the overall approach. The corpus results from an empirical study, where about 27 subjects had to give in-advance route descriptions towards a designated goal, while operating the 


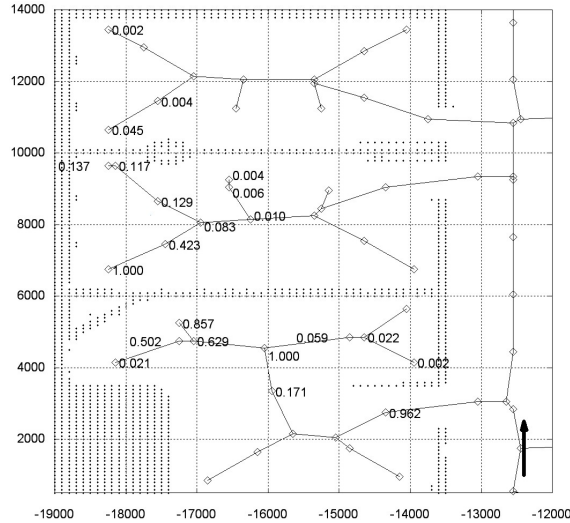

(a) The places to the front left.

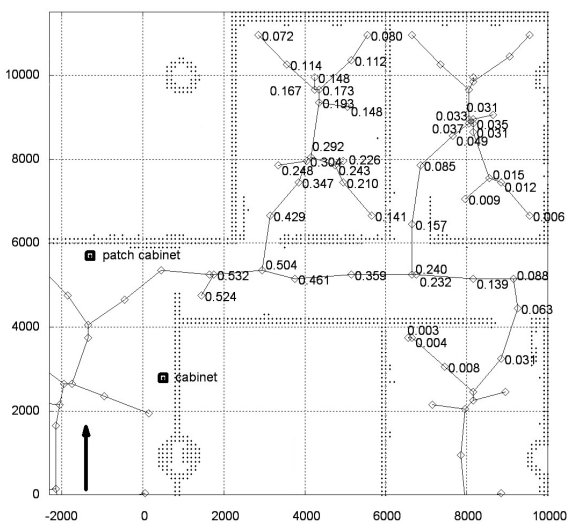

(b) Pass through the cabinet and the patch cabinet.

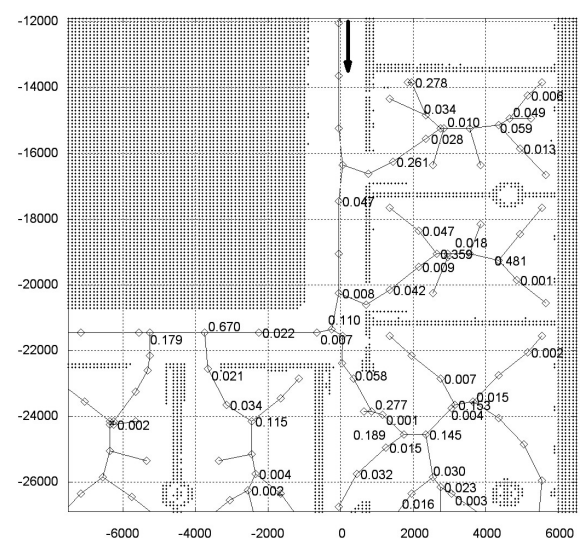

(c) Go until the 4th. junction to the left.

Fig. 2. Interpretation of spatial relations "NaturalLanguageDirection", "Through" and "Until" via fuzzy functions. The current pose of the agent i.e. the ego is depicted by a bold arrow. Prominent places acting as relatum are labeled by their fuzzy ratings.

autonomous wheelchair Rolland. See [3] and [18] for a brief discussion of this study. In both of the following examples that are translated from German to English, the subjects describe the way from their current pose to the so-called Stugaraum.

i) ...I have to turn around...once...180 degrees ${ }^{1}$...drive through the door...turn right...and pass through the connecting door...then turn right once again...then a quite long time straight ahead...pass the elevators...pass the main stairs...and then we arrive at the Stugaraum...

ii) ...we turn once...180 degrees...leave the room...on the corridor we turn 90 degrees to the left...follow the corridor...through the glass door...after the glass door we take the first junction...turn 90 dregrees to the left...continue straight ahead...through a glass door...down the corridor...through a second glass door...and on the left side there is the Stugraum...

Considering the italic printed reorientation blocks, the bold printed routers and normal typeset controlmarks, one can verify the basic form of definition 3 against the given examples. In spite of this achievement, the inner structure of each single building block remains undeveloped. For this reason we have extended the basic formalization of coarse route descriptions. A first look on the resulting EBNF in table I reveals the overall structure.

As in definition 3, a CoarseRouteDescription is still defined as an ordered sequence of CoarseRouteSegmentDescription, each of which now comprises a ControllerOnRouteSegment, a RouterOnRouteSegment and an ActionAtEndOfRouteSegment. So that the controller and the router are capable to represent a landmark along, respectively at the end of the route segment, they are now defined via a SpatialRelation and an AttributedPlace. Whereas the controller can characterize a route segment also via a DistancePredicate e.g. "a quite

\footnotetext{
${ }^{1}$ Note that this example is somewhat unusual, due to the precisely given turning angles. The translation of these numerical values into the proper spatial relations, e.g. 180 degrees $\sim$ NatLangDir::Back, is part of the semantic speech analysis and outside the scope of this work.
}

long time straight ahead", the expressiveness of controllers and routers basically lies in the combination of spatial relations and attributed places. A simple example for such a combination is "through a glass door", in which the spatial relation "through" is a three-valued relation. As the first argument it takes the current pose of the agent called ego. The attributed place "glass door" forms the second argument and is called relatum in an abstract way. The last argument referent is the hypothetical pose that is reached when moving from ego "through" the relatum. The EBNF in table I gives an open list of spatial relations whose order is denoted by an attached superscript.

A special spatial relation is given by NaturalLanguageDirection. This frequently appearing relation can either be used as a binary relation e.g. "the door to my left", or as a ternary relation such as "the door left to the elevator". To take account for the fact that humans use directions in different levels of granularity, four kinds of NaturalLanguageDirection have been defined, each of a different resolution. They range from NatLangDir::2Clock which solely differentiates Left and Right to NatLangDir::12Clock in analogy to the directions pointed to by a watch-hand at clock hours.

Completing the formalization of coarse route descriptions, an attributed place represents a landmark that is referenced via a spatial relation. To stay general, the type and the domain of an attribute are left unspecified. A typical example that is used in our implementation (cf. sec. V) is "the third junction to the left", representing a place that has the property of being the third junction with a possible veer to the left w.r.t. a given ego.

\section{Processing of CoArse Route Descriptions}

This section starts with a specification of fuzzy functions that correspond to the spatial relations that most frequently appeared in the corpora. We then proceed with the development of an algorithm that inputs a formalized $C R D$ (cf. sec. IV) representing the current task description, a global route graph (cf. sec. III-C) which holds the available world knowledge, and the global pose of the system. By evaluating 
each fuzzy function that corresponds to the consecutively appearing spatial relations in the CRD, our algorithm builds up a search tree that is searched in a depth-first branch-andbound manner to determine the most probable goal pose of the route description.

\section{A. Spatial Relations as Fuzzy Functions}

Our approach introduced here treats the task of evaluating spatial relations as a judgement of how well at least two given points out of the global workspace description can be correlated via the spatial relation to be analyzed. We chose fuzzy functions mapping to the domain $[0 . .1]$ because of the intrinsic coarse nature of natural language information.

1) Natural Language Direction: When humans use directional prepositions in natural language, they use them either as binary or ternary spatial relation. In this example we describe the binary case, in which the agent references a relatum from its current pose ego. As described in section IV, one uses natural language directions within different levels of granularity. Our illustration in Fig. 2(a) addresses the direction "FrontLeft" out of an eight-valued set of directions i.e. $<$ NatLangDir::8Clock $>$ (cf. table I). We start by computing the angle $\alpha$ between i) the straight line that connects ego with relatum and ii) the vector that is based in the position of ego and aligned to the current heading of ego. Taking the most compatible angle $\beta$ for the given direction "FrontLeft", which is $p i / 4$ w.r.t. the eight-valued level of granularity, and the normalizing constant $c$, we now compute the fuzzy rating of the spatial relation "FrontLeft" as can be seen in (4).

$$
F R_{N a t \text { LangDir }}(\text { ego, relatum })=e^{-\frac{1}{2}\left(\frac{|\alpha-\beta|}{c}\right)^{2}}
$$

2) Passing Through Given Landmarks: The ternary spatial relation "Through" takes as its first argument the current pose of the agent called ego. In our example the second argument is given by the two landmarks $l m_{1}=$ cabinet and $l m_{2}=$ patch cabinet, both labeled as relatum. The third argument referent is the global place we want to reach by passing from ego through relatum. This situation is illustrated in Fig. 2(b). Starting from ego depicted by the bold arrow, we choose an arbitrary relatum, that is to be evaluated by the spatial relation, in the neighborhood of ego. In a first step we apply a standard graph search algorithm to compute the route $\rho$ from the closest place nearby ego to relatum. If $\rho$ does not exist, we judge relatum with the lowest possible value 0 . Otherwise we continue with the computation of a circular region $\chi$ with diameter $d_{\chi}=\left\|l \vec{m}_{2}-l \vec{m}_{1}\right\|$ such that the centers of the landmarks lie on $\chi$ 's circumference. The mentioned circle $\chi$ now segments $\rho$ into the parts before, inside and after $\chi$. Taking the length $\lambda$ of $\rho$ after $\chi$, and the normalizing constant $c$, we now compute the fuzzy rating of the spatial relation "Through" as can be seen in (5).

$$
F R_{\text {Through }}(\text { ego, referent, relatum })=e^{-\frac{\lambda}{c}}
$$

The fact that fuzzy ratings decrease for places that are farther away from $\chi$ models the typical nature of human route de- scriptions in that single parts apply to the direct neighborhood of referenced landmarks.

3) Going Until Indexed Junction: The binary spatial relation "Until" takes as its first argument the current pose of the agent called ego. In our example the second argument relatum is given by the place until which we want to go from ego, i.e. the $i=4_{t h}$ place with a junction to the left. In order to look for an indexed place with a given attribute, we first have to define the fuzzy rating for the existence of that attribute in a given place $\varnothing$. Therefore we model a junction to a given natural language direction in analogy to the binary direction relation in section V-A.1, however with a different computation of the angle $\alpha$. In this case the angle $\alpha$ is computed as the angle between two route segments sharing the common place $\varnothing$. We now start with the computation of the route $\rho$ from the closest place nearby ego to relatum. Interpreting $\rho$ as an ordered set of places $\left\{p_{0}, p_{1}, \ldots, p_{n}\right\}$, we compute the ordered set of fuzzy ratings $F R(\rho)=\left\{f r_{1}, f r_{2}, \ldots, f r_{n}\right\}$, which represent the judgements that there exists a junction to the left in the corresponding places $p_{i}$. Considering the $j=\left(\begin{array}{c}n \\ i\end{array}\right)$ possible combinations to find in $p_{n}$ the $i_{t h}$ place with a junction to the left, we can now compute $\left\{\mathcal{A}_{1}, \mathcal{A}_{2}, \ldots, \mathcal{A}_{j}\right\}$ as the $\left(\begin{array}{c}n \\ i\end{array}\right)$-subsets of $F R(\rho)$ (cf. [19]), yielding the overall fuzzy rating for the spatial relation "Until" as can be seen in (6).

$$
F R_{U n t i l}(\text { ego, relatum })=\sum_{k=1}^{j} \prod_{l=1}^{n}\left\{\begin{array}{r}
f r_{l}: f r_{l} \in \mathcal{A}_{k} \\
1-f r_{l}: f r_{l} \notin \mathcal{A}_{k}
\end{array}\right.
$$

\section{B. Evaluation of Coarse Route Descriptions via Search Trees}

For the evaluation of coarse route descriptions, i.e. the calculation of the most likely target pose, we utilize a search tree the nodes of which represent fuzzy rated places that result from the evaluation of single CRD-elements. Fig. 3 illustrates the construction of the search tree. Considering a single node as a compound of a pose and its fuzzy rating or score, the root $p 1$ is given by the initial pose of the agent along with the highest possible score 1 . Taking the first spatial relation $S R_{1}$ that appears in the ordered list of coarse route segment descriptions, one chooses the first candidate pose $p 2$

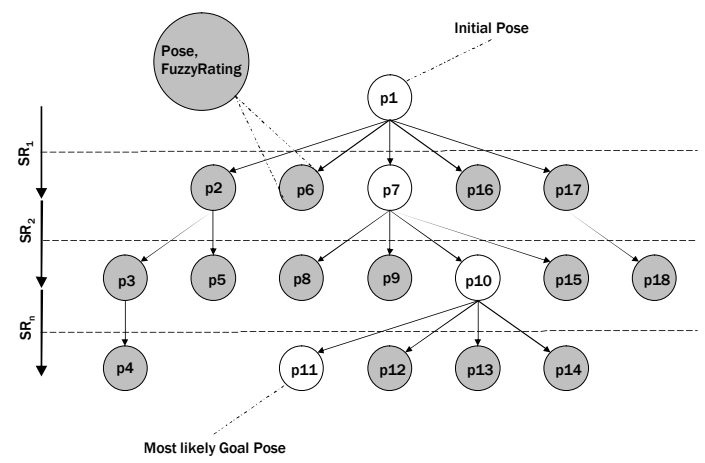

Fig. 3. Evaluation of a coarse route description via depth-first, branch-andbound search. 
that acts as the relatum for the fuzzy function associated with $S R_{1}$. After calculating $p 2$ 's own score, it is multiplied by the score of the parent node $p 1$. This mechanism propagates the uncertainty of former fuzzy ratings down to lower levels of the search tree. The algorithm now recursively continues in a depth-first branch-and-bound manner with the evaluation of the following spatial relations. In order to keep the size of the search tree small, we apply two different bound-criteria. First we do not construct nodes from the evaluation of a single spatial relation if the resulting score is lower than a given threshold $c_{1}$. Second we stop the evaluation at a given node if its cumulative score is already lower than the highest score of a leaf resulting from the evaluation of the final spatial relation. In addition to these rules, we only address candidates for the relatum of the spatial relation to be analyzed that are closer to the current ego than a given distance $c_{2}$.

\section{CONCLUSION}

In this work we have addressed the formalization of coarse qualitative route description in order to apply them to the mobile robot navigation domain. It has been shown that the involved spatial relations can be regarded as the key-elements in understanding route descriptions. By interpreting them with the help of fuzzy functions, we were able to derive the most probable target pose from a given route description. As an example of our real world experiments (cf. Fig. 4 for an illustration), the following instruction has been formalized, evaluated and executed in an office-like environment.

...go to the elevators area and continue until the first junction to the left...turn left there and pass through the cabinet and the patch cabinet...then continue until the second door to the left...turn left, go through the door and stop...

The findings presented so far bridge a gap between low-level speech recognition and ontological language understanding on the one hand and path planning for mobile robots on the other hand. Upcoming work has to focus on the investigation of further spatial relations in order to interpret the wide choice of natural language route descriptions.

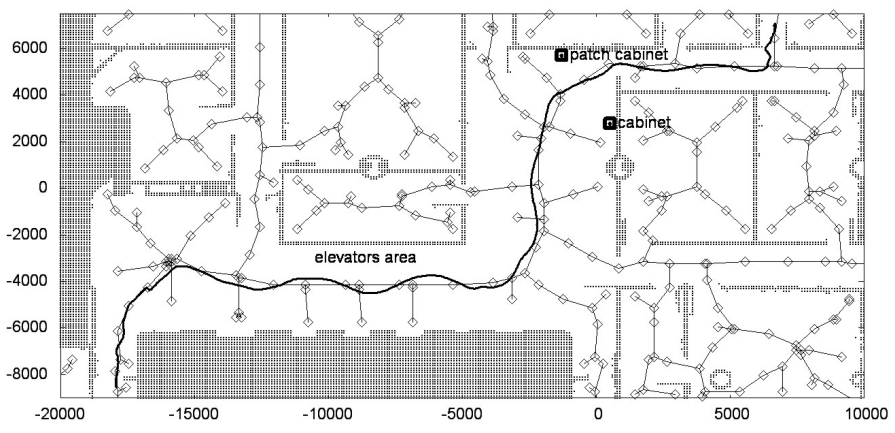

Fig. 4. Test run as estimated by the Monte Carlo Localizer: Starting at the corridor to the lower left, going via the elevators area, passing through the cabinets and finishing in the office to the upper right. Note that the depicted trajectory results from a real world experiment that has been conducted with an autonmomously moving robot, instructed by spoken route descriptions (cf. [20] for an illustrative video).

\section{ACKNOWLEDGMENT}

This work has been funded by the Deutsche Forschungsgemeinschaft (DFG) in context of the Sonderforschungsbereich/Transregio 8 Spatial Cognition.

\section{REFERENCES}

[1] C. Mandel, K. Hübner, and T. Vierhuff, "Towards an autonomous wheelchair: Cognitive aspects in service robotics," in Proceedings of Towards Autonomous Robotic Systems (TAROS), 2005.

[2] R. Müller, A. Lankenau, A. Musto, K. Stein, and A. Eisenkolb, "Coarse qualitative descriptions in robot navigation," in Spatial Cognition II, Lecture Notes in Artificial Intelligence, 2000.

[3] I1-[OntoSpace]. (2004) Human-robot interaction. SFB/TR8 Spatial Cognition, University of Bremen. [Online]. Available: http://134.102.58.154/human-robot.html

[4] S. Thrun, "Towards a framework for human-robot interaction," Human Computer Interaction, 2003.

[5] A. Knoll, B. Hildebrandt, and J. Zhang, "Instructing cooperating assembly robots through situated dialogues in natural language," in Proceedings of the IEEE International Conference on Robotics and Automation (ICRA), 1997.

[6] T. Kyriacou, G. Bugmann, and S. Lauria, "Vision-based urban navigation procedures for verbally instructed robots." [Online]. Available: citeseer.ist.psu.edu/651267.html

[7] G. Chronis and M. Skubic, "Robot navigation using qualitative landmark states from sketched route maps," in Proceedings of the IEEE Intl. Conf. on Robotics and Automation (ICRA), 2004.

[8] A. Lankenau and T. Röfer, "A safe and versatile mobility assistant," IEEE Robotics and Automation Magazine, vol. 8, no. 1, pp. 29-37, 2001.

[9] D. Fox, W. Burgard, and S. Thrun, "The dynamic window approach to collision avoidance, Tech. Rep. IAI-TR-95-13, 1 1995. [Online]. Available: citeseer.ist.psu.edu/fox 97dynamic.html

[10] J. Borenstein and Y. Koren, "Real-time obstacle avoidance for fast mobile robots," IEEE Transactions on Systems, Man, and Cybernetics, vol. 19, no. 5, pp. 1179-1187, - 1989. [Online]. Available: citeseer.ist.psu.edu/article/borenstein90realtime.html

[11] J. Minguez and L. Montano, "Nearness diagram (nd) navigation: Collision avoidance in troublesome scenarios," IEEE Transactions on Robotics and Automation, vol. 20, no. 1, 022004.

[12] — " "The ego-kinodynamic space (ekds): Shape, kinematics and dynamics of the vehicle," in Proceedings of the IEEE Intl. Conf. on Intelligent Robots and Systems (IROS), 2003.

[13] J.-H. Hwang, R. C. Arkin, and D.-S. Kwon, "Mobile robots at your fingertip: Bezier curve on-line trajectory generation for supervisory control," in Proceedings of the IEEE Intl. Conf. on Intelligent Robots and Systems (IROS), 2003.

[14] T. Röfer and M. Jüngel, "Vision-based fast and reactive monte-carlo localization," in Proceedings of the IEEE International Conference on Robotics and Automation (ICRA), 2003.

[15] S. Lenser and M. Veloso, "Sensor resetting localization for poorly modeled mobile robots," in Proceedings of the IEEE International Conference on Robotics and Automation (ICRA), 2000.

[16] B. Krieg-Brückner, U. Frese, K. Lüttich, C. Mandel, T. Mossakowski, and R. Ross, "Specification of an ontology for route graphs," in Spatial Cognition IV, Lecture Notes in Artificial Intelligence, 2004.

[17] S. Werner, B. Krieg-Brückner, and T. Herrmann, "Modelling navigational knowledge by route graphs," in Spatial Cognition II, Lecture Notes in Artificial Intelligence, 2000.

[18] S. Hui and T. Tenbrink, "Telling rolland where to go: HRI dialogues on route navigation." in Proc. Workshop on Spatial Language and Dialogue (5th Workshop on Language and Space), 2005.

[19] A. Nijenhuis and H. Wilf, Combinatorial Algorithms. Academic Press, 1978.

[20] A1-[RoboMap] and I3-[SharC]. (2006) Interpretation and execution of verbal route descriptions by the autonomous wheelchair rolland. SFB/TR8 Spatial Cognition, University of Bremen. [Online]. Available: http://www.informatik.uni-bremen.de/rolland/videos/Interpretation_of_ Coarse_Route_Description_08_06_06.mpg 